CASE REPORT

\title{
Multiple brain abscesses in a patient with bilateral pulmonary arteriovenous malformations and immunoglobulin deficiency
}

\author{
K-C Tse, G C Ooi, A Wu, P L Ho, S K Ip, M H Jim, Y M Lam, Y W Fan, W K Tso, K W Tsang
}

Postgrad Med J 2003;79:597-599

A 34 year old Chinese man presented with grand mal seizures complicating multiple brain abscesses caused by mixed oral flora. Because of persistent hypoxaemia contrast spiral thoracic computed tomography was done, which revealed bilateral pulmonary arteriovenous malformations (PAVMs). Concomitant lgA and $\lg G$ subclass deficiency was also found. The combination of these two conditions appears to have predisposed this patient to presumably paradoxical septic embolism. The patient's cerebral condition responded to postoperative antibiotic treatment and he eventually received selective coil embolisation of right lower lobe PAVMs, which relieved his hypoxaemia and dyspnoea.

$\mathrm{P}$ ulmonary arteriovenous malformation (PAVM) is a rare congenital vascular anomaly, associated with OslerWeber-Rendu syndrome, initially described in 1897, and is due to formation of abnormal communications between pulmonary arteries and veins. ${ }^{12}$ Clinical manifestations can be subtle and this condition may remain undiagnosed until complications arise in adulthood. We describe a patient who presented with seizures secondary to multiple brain abscesses, which were presumably caused by paradoxical embolism via PAVM and predisposed by IgA and IgG deficiency.

\section{CASE REPORT}

A 34 year old southern Chinese man presented to our accident and emergency department with his first grand mal seizure in April 2002. There was no history of recent viral illness or systematic symptoms. A history of recurrent right foot cellulitis and benign colonic tumour, which was resected in his teenage years, was noted. His older brother suffered from an atrial septal defect, which was repaired but this was later complicated by infective endocarditis. There was no recent history of dental or other surgical procedures. Physical examination revealed a fever of $38.2^{\circ} \mathrm{C}$ and finger clubbing. His oxygen saturation was $98 \%$ on $4 \mathrm{l} / \mathrm{min}$ oxygen therapy via a nasal cannula. There were no focal neurological signs. The patient became conscious and orientated on arrival to our accident and emergency department. Cardiorespiratory examination was normal. Urgent computed tomography of the brain showed multiple hypodense lesions with rim enhancement and oedema consistent with multiple brain abscesses in the left temporal and occipital lobes (fig 1), which required urgent burr hole operation. Culture of aspirated pus yielded polymicrobial growth of Actinomyces odontolyticus, Streptococcus milleri and Fusobacterium species, although blood cultures were negative. Examination of the ear, nose, and throat and oral cavity was normal. There was no mucosal or cutaneous telangiectasia. Transthoracic and transoesophageal echocardiography showed normal heart valves and no intracardiac shunting.

A six week course of intravenous penicillin $G$ and metronidazole was planned. The patient responded rapidly and became apyrexial within 48 hours after initial admission. There was no recurrence of seizures. However, there was persistent hypoxaemia with a oxygen saturation between $88 \%$ and $92 \%$, arterial oxygen pressure of $8.3 \mathrm{kPa}$, and arterial carbon dioxide pressure of $5.0 \mathrm{kPa}$ while breathing room air. Chest radiography showed only prominent vascular shadows but was otherwise unremarkable (fig 2). Contrast enhanced spiral computed tomography of the thorax was, therefore, performed which revealed no evidence of pulmonary embolism. However, there were multiple enhancing serpinginous structures in both lung bases suggestive of small vascular masses (fig 3A and B). Magnetic resonance angiography (MRA) confirmed that these were multiple PAVMs with arterial supply from dilated descending pulmonary arteries with drainage into the pulmonary veins in both lung bases (fig 4). There were more PAVMs in the right lower lobe than in the contralateral lobe. The presence of this shunt was also confirmed by right heart catheterisation, which showed PAVMs in the right lower lobe and anterior segment of left lower lobe.

Initial blood tests revealed normal total and differential leucocyte counts, and renal and liver function parameters. Serum IgG was $8.79 \mathrm{~g} / \mathrm{l}$ (normal: 8.19-17.25 g/l), IgA $<0.067 \mathrm{~g} / \mathrm{l}$ (normal: $0.70-3.86 \mathrm{~g} / \mathrm{l}$ ), and $\operatorname{IgM} 0.68 \mathrm{~g} / \mathrm{l}$ (normal: $0.55-3.07 \mathrm{~g} / \mathrm{l}$ ). IgG subclass analysis revealed $\operatorname{IgG}_{1}$ of $2.80 \mathrm{~g} / \mathrm{l}$ (normal: $4.22-12.92 \mathrm{~g} / \mathrm{l}$ ), $\mathrm{IgG}_{2}$ of $0.70 \mathrm{~g} / \mathrm{l}$ (normal: 1.17-7.47 g/l), $\mathrm{IgG}_{3}$ of $0.33 \mathrm{~g} / \mathrm{l}$ (normal: $\left.0.41-1.29 \mathrm{~g} / \mathrm{l}\right)$, and $\mathrm{IgG}_{4}$ of $0.09 \mathrm{~g} / \mathrm{l}$ (normal: 0-2.91 g/l). However, there was no evidence of associated autoimmune diseases with normal antinuclear antibody, C3, and C4. Serum immunoelectrophoresis was also normal, and there was no evidence of underlying haematological malignancy. Serology for HIV was negative.

The patient made an uneventful neurological recovery, and completed his course of intravenous antibiotics uneventfully. In view of the larger and more numerous PAVMS in the right lower lobe, selective coil embolisation of these right lower lobe PAVMs was performed via right common femoral vein puncture under local anaesthesia using 3-6 mm fibred platinum coil vortex 0.35 (Target Therapeutics, Boston Scientific Corporation, Freemont, USA) and $3 \mathrm{~mm}$ fibred platinum coil 0.035 type (Target Vascular, Boston Scientific Cork Ltd, Cork, Ireland). The left lower lobe PAVMs were not embolised. At the time of writing, six months after his

Abbreviations: MRA, magnetic resonance angiography; PAVM, pulmonary arteriovenous malformation 


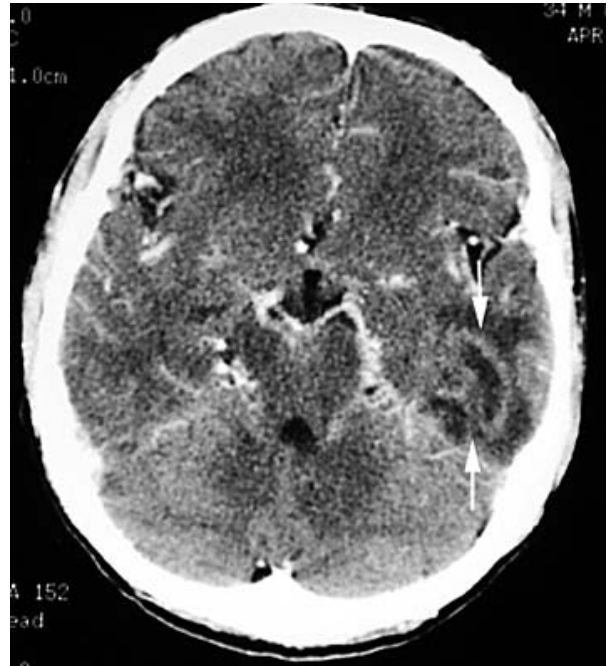

Figure 1 Contrast enhanced computed tomogram of the brain showing a hypodense nodule with rim enhancement and marked surrounding vasogenic oedema consistent with brain abscess in the left temporal lobe.

embolisation procedures, this patient has remained asymptomatic neurologically, and has a stable oxygen saturation of 96\%-98\% (when breathing room air). The patient is undergoing regular intravenous immunoglobulin replacement under the care of an immunologist.

\section{DISCUSSION}

Our patient presented with an interesting array of events, which could ultimately be interpreted as the complications of PAVMs and immunoglobulin deficiencies. The occurrence of multiple brain abscesses, caused by oral flora, suggests that paradoxical embolisms could have occurred as the underlying key mechanism for the development of septic embolism, presumably aggravated by underlying immunoglobulin deficiencies. Paradoxical embolism is a well known complication of PAVMs. Other features of PAVMs include hypoxaemia, dyspnoea, clubbing, cyanosis, polycythaemia, haemoptysis, cerebral infarction, high output cardiac failure, and abnormal chest radiology. The cause of hypoxaemia in patients with PAVMs is due to persistent shunting of deoxygenated blood to the systemic circulation with a similar mechanism to cyanotic congenital heart diseases, except that this shunt is

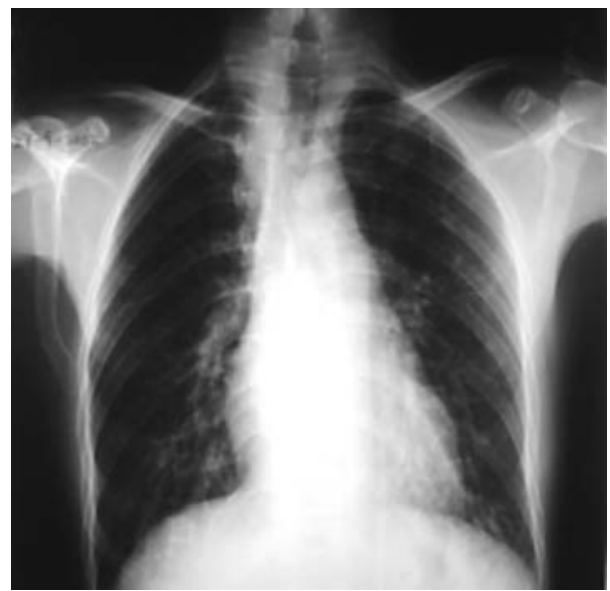

Figure 2 Routine chest radiograph showing increased vascular markings in the lower lung zones.

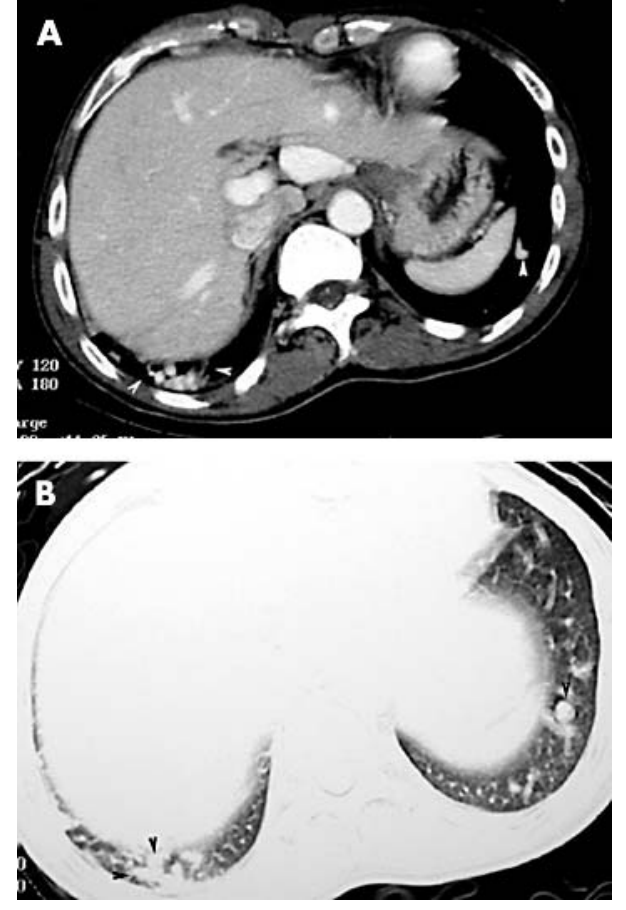

Figure 3 Contrast enhanced computed tomograms in (A) mediastinal and (B) lung windows showing serpinginous (arrows) and rounded (arrowheads) structures that enhance in the lung bases. These are suggestive of small but multiple PAVMS.

extracardiac in location. Chest radiographs may be abnormal, but changes are frequently non-specific, and the diagnosis may be made incidentally from computed tomography examination particularly in small PAVMs such as found in our case. ${ }^{3}$ While pulmonary angiography remains the traditional gold standard, MRA is increasingly used for the noninvasive diagnosis of PAVMs, with contrast echocardiography useful in detecting right to left shunting. ${ }^{4}$ Although PAVMs are rare and therefore likely to be under-diagnosed, they are present in $24 \%$ of patients with Osler-Weber-Rendu syndrome. ${ }^{5}$ Features of associated Osler-Weber-Rendu syndrome, namely the presence of telangiectasia, should therefore be sought in patients with PAVMs, and if present the family should be screened.

Our case is of particular clinical and academic interest as IgA and IgG subclass deficiency exists concomitantly. Selective IgA deficiency is the most common of the immunodeficiency disorders and affects one in 500 indivi-

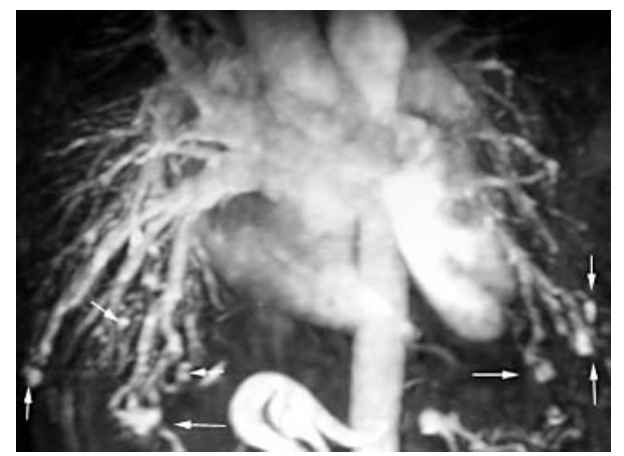

Figure 4 MRA of the thorax showing bilateral multiple lower lobe PAVMs (arrows). The right lower lobe is more severely involved than the left. 


\section{SUMMARY POINTS}

- Presentations of PAVMs are frequently subtle and should be suspected in a patient with apparently unexplained hypoxaemia.

- The gold standard for diagnosis is by pulmonary angiography and treatment with coil embolisation is effective especially for multiple PAVMs.

- There is a potential association with primary immunoglobulin deficiency.

duals. The majority of these individuals remain asymptomatic, but some patients might develop sinopulmonary infections, chronic diarrhoeal illness, and autoimmune phenomena. Selective IgA deficiency rarely leads to destructive lung disease, unless there is an associated IgG subclass deficiency, especially of $\mathrm{IgG}_{2}$. In a recent study, 13 of 56 bronchiectasis patients had deficiencies of one or more IgG subclass, ${ }^{7}$ although our patient did not have bronchiectasis on computed tomography. Only one other similar case has been reported previously in the literature, which described a middle aged man with telangiectasia of the lips, tongue and PAVMs, and hypogammaglobulinaemia complicated by brain abscesses. ${ }^{8}$ Our case is, however, unique as there was a complete absence of cutaneous telangiectasis. A possible association between underlying immunological defects and the development of vascular malformations has recently been proposed, in view of the concomitance of these conditions among patients with ataxia telangiectasia and AIDS. ${ }^{9}$ However, the exact mechanism(s) underlying this potential causal relationship is obscure and clearly needs further evaluation. Patients with immunoglobulin deficiency need to be followed up regularly as some of them ultimately develop a common variable immunodeficiency. ${ }^{11}$ Maintenance prophylactic antibiotic or intravenous IgG replacement is beneficial, but there is a risk of anaphylactic reaction to the latter due to the inherent presence of anti-IgA in some of these patients. ${ }^{12}$

\section{Authors' affiliations}

K-C Tse, A Wu, S K Ip, M H Jim, Y M Lam, K W Tsang, Department of Medicine, University of Hong Kong, Queen Mary Hospital, Hong Kong, China

G C Ooi, W K Tso, Department of Radiology

P L Ho, Department of Clinical Microbiology

Y W Fan, Department of Neurosurgery

Correspondence to: Dr Kenneth W Tsang, Department of Medicine, Queen Mary Hospital, 102 Pokfulam Road, Hong Kong SAR, China; kwttsang@hku.hk

Submitted 12 December 2002

Accepted 15 February 2003

\section{REFERENCES}

1 Churton T. Multiple aneurysms of the pulmonary artery. BMJ 1897;i:1223.

2 Khurshid I, Downie GH. Pulmonary arteriovenous malformation. Postgrad Med J 2002;78:191-7.

3 Quaden C, Ghaye B, Dondelinger RF, et al. Multiple pulmonary arteriovenous malformations. Lancet 2002;359:1998.

4 Barzilai B, Waggoner AD, Spessert C, et al. Two-dimensional contrast echocardiography in the detection and follow-up of congenital pulmonary arteriovenous malformations. Am J Cardiol 1991;68:1507.

5 Kjeldsen AD, Oxhoj $\mathrm{H}$, Andersen PE, et al. Prevalence of pulmonary arteriovenous malformations (PAVMs) and occurrence of neurological symptoms in patients with hereditary haemorrhagic telangiectasia (HHT). J Intern Med 2000;248:255-62.

6 Waight DJ, Hijazi ZM. Pulmonary arteriovenous malformations: transcatheter embolization options. Catheter Cardiovasc Interv 2000;50:52-3.

7 Stead A, Douglas JG, Broadfoot CJ, et al. Humoral immunity and bronchiectasis. Clin Exp Immunol 2002;130:325-30.

8 Couderc L, Caubarrere I, Oksenlendler E, et al. Vascular malformations and hypogammaglobulinaemia. Lancet 1987;i:385.

9 Waldmann TA. Ataxia-telangiectasia: a multisystem hereditary disease with immunodeficiency, impaired organ maturation, X-ray hypersensitivity, and a high incidence of neoplasia. Ann Intern Med 1983;99:367-79.

10 Fallon T, Abell E, Kingsley L, et al. Telangiectases [corrected] of the anterior chest in homosexual man. Ann Intern Med 1986;105:679-82.

11 Espanol T, Catala M, Hernandez M, et al. Development of a common variable immunodeficiency in IgA-deficient patients. Clin Immunol Immunopathol 1996;80:333.

12 Buckley RH, Schiff RI. The use of intravenous immune globulin in immunodeficiency diseases. N Engl J Med 1991;325:110. 